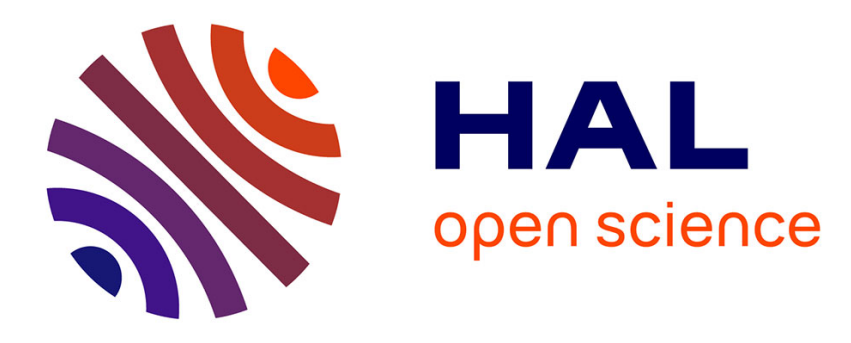

\title{
An Occlusion Calculus Based on an Interval Algebra
}

Paulo E Santos, Gérard Ligozat, Marjan Safi-Samghabadi

\section{To cite this version:}

Paulo E Santos, Gérard Ligozat, Marjan Safi-Samghabadi. An Occlusion Calculus Based on an Interval Algebra. 4th Brazilian Conference on Intelligent Systems (BRACIS 2015), Nov 2015, Nadal, Brazil. pp.128 - 133, 10.1109/BRACIS.2015.12 . hal-01433863

\section{HAL Id: hal-01433863 https://hal.science/hal-01433863}

Submitted on 13 Jan 2017

HAL is a multi-disciplinary open access archive for the deposit and dissemination of scientific research documents, whether they are published or not. The documents may come from teaching and research institutions in France or abroad, or from public or private research centers.
L'archive ouverte pluridisciplinaire HAL, est destinée au dépôt et à la diffusion de documents scientifiques de niveau recherche, publiés ou non, émanant des établissements d'enseignement et de recherche français ou étrangers, des laboratoires publics ou privés. 


\section{An Occlusion Calculus based on an Interval Algebra}

\author{
Paulo E. Santos \\ Centro Universitário da FEI, \\ S. Paulo, Brazil
}

\author{
Gérard Ligozat \\ Université Paris-Sud, \\ Paris, France
}

\author{
Marjan Safi-Samghabadi \\ Alzahra State University, \\ Tehran, Iran
}

\begin{abstract}
This paper introduces a new qualitative spatial reasoning formalism, called Interval Occlusion Calculus (IOC), that takes into account multiple viewpoints of a scene. This formalism extends Allen's Algebra by including an interval-based definition for spatial occlusion. We prove that IOC is a relation algebra and show complexity results for this formalism.
\end{abstract}

\section{INTRODUCTION}

Qualitative spatio-temporal reasoning (QSTR) is a subfield of knowledge representation in Artificial Intelligence whose aim is to develop qualitative representations (and reasoning methods) involving spatial, temporal or spatio-temporal aspects of reasoning, such as spatial regions, directions, temporal intervals, spatio-temporal histories, among others [1], [2].

This paper falls within a small subset of QSTR formalisms that take into account the observer's viewpoint explicitly within their theories (e.g. [3]). Most previous works on viewpointdependent QSTR calculi, however, assume only the representation and inferences related to the spatial perception of a single agent, leaving aside issues related to reasoning about multiple viewpoints in a scene. In order to cope with this issue, the present paper reports the development of a multiple-view spatial reasoning system, based on Allen's Interval Algebra [4]. This formalism, which we call Interval Occlusion Calculus (IOC), represents explicitly various distinct viewpoints whereby the notions of object occlusion and knowledge sharing between agents play an important role.

Most of the QSTR formalisms that consider an observer viewpoint have spatial occlusion (or motion parallax) as a key aspect of their ontology. Spatial occlusion occurs when an object interposes between another with respect to an observer's viewpoint; it is considered as one of the cues used by the human perceptual system to construct a 3D interpretation of the visual world [3]. Perhaps the first qualitative formalisation of spatial occlusion was proposed in [5] where a set of axioms is designed to constrain a point-based notion of occlusion. Assuming 2D convex objects, rather than points, [6] proposes the Lines-of-Sight calculus that represents the relative positions between pairs of bodies as seen from a viewpoint. Based on this idea, the Region Occlusion Calculus [3] defines occlusion and image parallax within a mereotopological theory. The work described in [7] proposes a modal logic on binary relations representing visibility. In contrast, [8] proposes a set of relations making explicit the distinctions of whether the observed objects are fragmented or not, and whether the occluder is a moving object or part of the background.

The works cited above, however, do not take into account the possible interactions between multiple viewpoints. In the current scientific literature we can find only a few papers describing the use of spatial reasoning in situations where multiple (distinct) viewpoints are needed. [9] defines a distributed notion of spatial information processing, where the various procedures for inference and analyses of spatial knowledge (such as line segmentation, the identification of relations between points or regions) are distributed over distinct agents. Another approach is proposed in [10], where the solutions generated by multiple agents are integrated via a collaborative decision process, in which a number of qualitative spatial constraints should be satisfied. Closer to the work described in this paper, [11] combines the cardinal location of objects as observed by multiple viewpoints by means of a constraint satisfaction procedure.

In contrast to the work overviewed in this section, the present paper develops a QSTR theory about occlusion and multiple viewpoints upon a relation algebra. This allows for the formal definition of constraint satisfaction and provides a clear view of the complexity of reasoning.

\section{A Point Occlusion Calculus}

In this section we introduce a pointwise definition for an occlusion calculus that we call Point Occlusion Calculus (POC), that illustrates the contents of this paper.

Let $A$ and $B$ be a pair of distinct points, as shown in Figure 1. Consider a point-wise agent in the plane outside the line $\overline{A B}$. In Point Occlusion Calculus, if this agent is located on the right-hand side of $\overrightarrow{A B}$, it can assert that $A<B$ ( $A$ precedes $B$ ). If it is located in the opposite half plane, it asserts that $A>B(A$ is preceded by $B)$. There are two further possibilities if the observer is located on the line $\overline{A B}$ (but not in between $A$ and $B$ ): either it can see $A$, which totally occludes $B\left(A c^{+} B\right)$, or it can see $B$, but not $A$ ( $A$ is totally occluded by $B: A c^{-} B$ ). If the observer is located on the line $\overline{A B}$, but in between $A$ and $B$, it can observe $A<B$ if oriented towards the left hand side of $\overrightarrow{A B}$ (i.e. towards the top of the page in Fig. 1), or $A>B$ if oriented in the opposite direction.

It is worth pointing out that the diagram in Fig. 1 shows what an observer can see, and not what it actually perceives (i.e. an observer could be located on the right-hand side of $\overrightarrow{A B}$ but oriented toward the bottom of the page, thus neither seen $A$ nor $B$ ).

Together with equality (denoted by $e q$ ), this makes a set of basic relations $\left\{\langle\rangle,, c^{+}, c^{-}, e q\right\}$ of the Point Occlusion Calculus (POC). In the corresponding algebra, inversion exchanges $\left\langle\right.$ and $>, c^{+}$and $c^{-}$, and leaves $e q$ fixed. POC allows an agent to describe the relative views with respect to two distinct punctual objects. The remainder of this paper extends POC to two dimensions using Allen's Interval Algebra. 


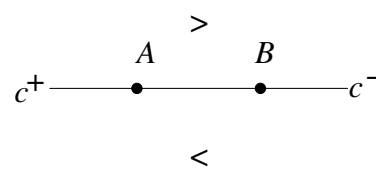

Fig. 1. The point occlusion calculus.

In order to introduce the idea of multiple viewpoints in Allen's relations we have to consider the definitions below.

\section{Allen's Interval Algebra}

Allen's interval algebra [4] is defined by a set of 13 jointlyexhaustive and pairwise-disjoint base relations representing the possible relations between pairs of intervals. Given two intervals, $x$ and $y$, the Allen's relations are shown in Figure 2.

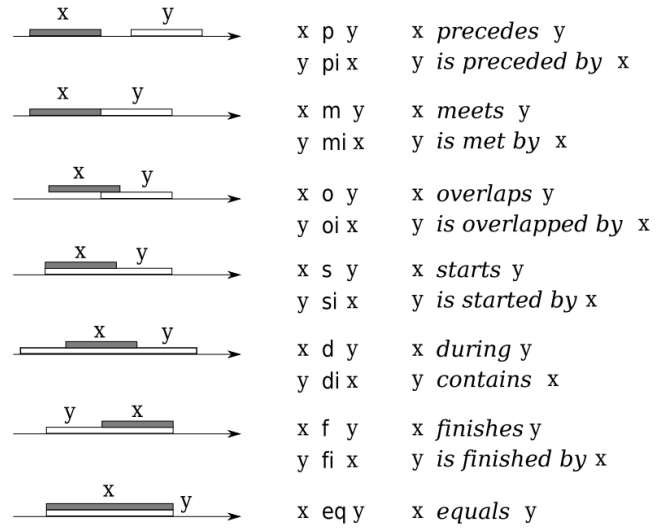

Fig. 2. Allen's Relations (adapted from [2]).

As presented in [2], consistency in a scenario described by a set of Allen's relations can be verified by enforcing algebraic closure (also known as Path Consistency) on a network of constraints. A constraint network is a pair $(N, C)$, where $N$ is the set of vertices (each vertex representing a domain element), and $C$ is a set of constraints (i.e. Allen basic relations).

Let $C(i, j) \in C$ be a constraint on a vertex $(i, j) \in N \times N$. The network $(N, C)$ is algebraically closed if for any triple $(i, j, k) \in N^{3}$ we have: $C(i, j) \subseteq(C(i, k) \circ C(k, j))$. Algebraic closure can be enforced by iterating

$$
C(i, j) \leftarrow C(i, j) \cap(C(i, k) \circ C(k, j)),
$$

as long as a change occurs. As the set of basic relations is finite, this process becomes stationary after a finite time.

\section{VIEWPOINTS WITHIN ALLEN'S RELATIONS}

We consider two kinds of entities living in the Euclidean plane: objects and observers. Both objects and observers are considered as rigid, convex, bodies. Objects can be identified by the 2D position of their centroids. Observers are represented as pairs $\Sigma_{i}=\left(x_{i}, \nu_{i}\right)$, where $x_{i}$ is a $2 \mathrm{D}$ position (the location of the observer's centroid) and $\nu_{i}$ is an unit vector representing the observer's orientation. In this paper we refer to an observer as a viewpoint using the variables $\Sigma_{i}(i \in \mathbb{N})$, thus a viewpoint variable has implicit the observer's location $\left(x_{i}\right)$ and its orientation $\left(\nu_{i}\right)$. As observers have an extended geometry, any observer is perceived as an object when located within the field of view of another viewpoint.

We define the field of view of a viewpoint $\Sigma$ as the half plane that includes $\Sigma$ and whose boundary line is perpendicular to $\Sigma$ 's direction (i.e. the observer's field of view is within the interval $\left[-90^{\circ},+90^{\circ}\right]$ from its direction of gaze $)^{1}$.

Objects in the world are perceived by observers by means of a function $\operatorname{image}(x, \Sigma)$ that maps an object $x$ to its image as seen from a viewpoint $\Sigma$. An image of an object from a viewpoint $\Sigma$ is defined as the set of projected half-lines originating at $\Sigma$, and contained in $\Sigma$ 's field of view, that intersects the object [3]. In a 2D world, images are intervals defined by the extreme points of observed objects projected on an observer's image plane. Note that this definition implies that the intervals representing observed objects are segments defined on a semi-circle. We also assume a clockwise orientation on these semi-circles. Figure 3 shows an example of the images of two circular objects seen from a viewpoint $\Sigma$, whereby the objects are perceived as the projections $a$ and $b$ (i.e., $a=\operatorname{image}(A, \Sigma)$ and $b=\operatorname{image}(B, \Sigma)$ ). In this example, the observer $\Sigma$ perceives segments $a$ and $b$ as $a$ preceding $b$, as defined in Section $\mathrm{V}$ below.

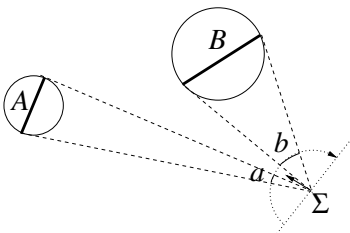

Fig. 3. Two circular objects $A$ and $B$ and a viewpoint $\Sigma$. The dashed lines represent the lines of sight and the dotted curves, the observer's field of view.

In this paper we assume that each viewpoint $\Sigma$ is capable of describing the relations between all the objects that are visible within its field of view. This description is given in terms of the relations on the intervals that represent the projections of observed objects on a semi-circle defining $\Sigma$ 's field of view (cf. Figure 3). Note that a viewpoint $\Sigma$ is an abbreviation for the observer's position and orientation, thus this description takes into account these two variables. This work assumes that the observations made from the viewpoints are available to the other agents by means of a message-passing procedure.

From the basic definitions described above, reasoning about occlusion from multiple viewpoints can be accomplished using an extension of Allen's interval calculus that takes into account the relationship between intervals in a two dimensional space. We call this extension the Interval Occlusion Calculus (IOC) as introduced in the next section.

\section{INTERVAL OCCLUSION CALCULUS}

Informally, the basic relations of the Interval Occlusion Calculus (IOC) can be understood as a description of the qualitative distinctions between the observation of pairs of objects, given the object's lines of sight, as shown in Figure 4. For instance, assuming that a viewpoint $\Sigma$ is oriented so that two objects $A$ and $B$ are within its field of view, and

\footnotetext{
${ }^{1}$ Choosing a $180^{\circ}$ field of view was arbitrary, assuming a narrower field of view would not cause much change in the theory presented.
} 
$a=\operatorname{image}(A, \Sigma)$ and $b=\operatorname{image}(B, \Sigma)$, if $\Sigma$ is standing on the region marked by $p$ (Fig. 4) then it could see that $a$ precedes $b$; if $\Sigma$ is, instead, located on top of one of the lines marked $m$, it could see that a meets $b$; a viewpoint on region $o^{+}$could see that a overlaps and is in front of $b$ and so forth. On the region between objects $A$ and $B$, a viewpoint oriented to the top of the page may see that $a$ precedes $b$; one oriented to the bottom of the page could see that $b$ precedes $a$. Note that a diagram similar to that shown in Fig. 4 can be defined for every pair of objects. The diagram in Figure 4 can be understood as a map with which agents can locate themselves with respect to the qualitative relations observed between pairs of objects. In this work we make the simplifying assumption that an observer occupies one and only one region of this map.

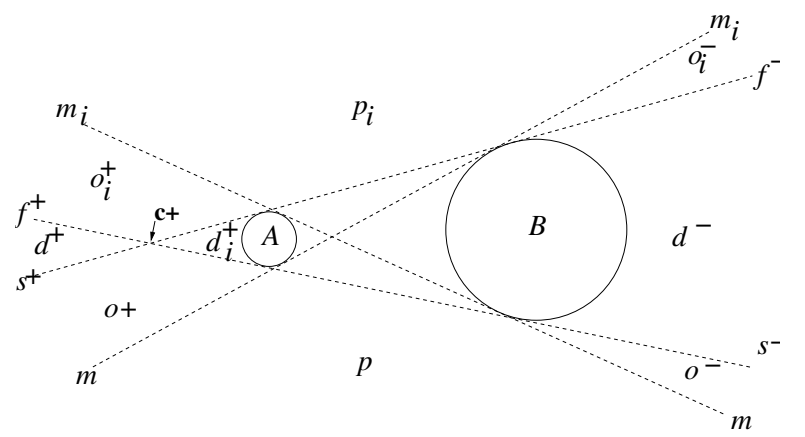

Fig. 4. The lines of sight between two objects $A$ and $B$ and the basic relations of the Interval Occlusion Calculus.

Let $L$ be a linear ordering. We define a layered interval $I$ as $I=\left(I_{a}, \ell\right)$, where $I_{a}$ is an interval, i.e. a pair $I_{a}=\left(x_{1}, x_{2}\right)$ of real numbers, where $x_{1}<x_{2}: x_{1}$ is the lower limit of $I$, and $x_{2}$ is its upper limit; and, $\ell$ is the layer of $I$, representing a linear ordering of intervals as observed from a viewpoint $(\ell \in L)$. We assume two functions on layered intervals: the function $\operatorname{ext}(I)$ that maps a layered interval $I$ to its extent (i.e. its upper and lower limits) and the function $\ell(I)$ that maps an interval $I$ to its layer.

In this work, every distinct interval has a distinct $\ell$ associated, and the ordering of the intervals with respect to $\ell$ represents their proximity to the observer (the closer an object is to the observer, the greater is its associated $\ell$ ). Given two distinct intervals $I$ and $J$ and a relation $r$, we use the following notation: $I r^{+} J$ iff $\ell(I)>\ell(J)\left(I r^{-} J\right.$ iff $\left.\ell(I)<\ell(J)\right)$.

With the definition of layered interval we can now formally define the relations of the Interval Occlusion Calculus in terms of Allen's interval relations. In the definitions below, the original Allen's relations are assigned a subscript $a$. Given two intervals $I$ and $J(I \neq J)$ representing the observation of two distinct objects from a viewpoint $\Sigma$, the IOC relations are defined as follows ${ }^{2}$ :

- $\quad I$ p $J$ : $\Sigma$, read as “ $I$ precedes $J$ from $\Sigma$ " iff $\operatorname{ext}(I) p_{a} \operatorname{ext}(J)$;

- $I m \quad J$ : $\Sigma$, read as " $I$ meets $J$ from $\Sigma$ " iff $\operatorname{ext}(I) m_{a} \operatorname{ext}(J)$;

- $\quad I o^{+} J: \Sigma$, read as " $I$ overlaps and is in front of $J$ from $\Sigma$ " iff $\operatorname{ext}(I) o_{a} \operatorname{ext}(J)$ and $\ell(I)>\ell(J)$;

\footnotetext{
${ }^{2}$ For brevity, we do not describe the inverse relations.
}

- $\quad I o^{-} J: \Sigma$, read as " $I$ overlaps $J$ and is $J$ is in front of $I$ from $\Sigma$ " iff $\operatorname{ext}(I) o_{a} \operatorname{ext}(J)$ and $\ell(I)<\ell(J)$;

$\circ o^{+}\left(o^{-}\right)$can also be read as "partially occludes" ("is partially occluded");

- $\quad I s^{+} J: \Sigma$, read as " $I$ starts and is in front of $J$ from $\Sigma$ " iff $\operatorname{ext}(I) s_{a} \operatorname{ext}(J)$ and $\ell(I)>\ell(J)$;

- $\quad I s^{-} J: \Sigma$, read as " $I$ starts $J$ and $J$ is in front of $I$ from $\Sigma$ " iff $\operatorname{ext}(I) s_{a} \operatorname{ext}(J)$ and $\ell(I)<\ell(J)$;

- $\quad I d^{+} J: \Sigma$, read as " $I$ is during and is in front of $J$ from $\Sigma$ " iff $\operatorname{ext}(I) d_{a} \operatorname{ext}(J)$ and $\ell(I)>\ell(J)$;

- $\quad I d^{-} J: \Sigma$, read as " $I$ is during $J$ and $J$ is in front of $I$ from $\Sigma$ " iff $\operatorname{ext}(I) d_{a} \operatorname{ext}(J)$ and $\ell(I)<\ell(J)$;

- $I f^{+} J: \Sigma$, read as " $I$ finishes and is in front of $J$ from $\Sigma$ " iff $\operatorname{ext}(I) f_{a} \operatorname{ext}(J)$ and $\ell(I)>\ell(J)$;

- $\quad I f^{-} J: \Sigma$, read as " $I$ finishes $J$ and $J$ is in front of $I$ from $\Sigma$ " iff $\operatorname{ext}(I) f_{a} \operatorname{ext}(J)$ and $\ell(I)<\ell(J)$;

- $I c^{+} J: \Sigma$, read as " $I$ coincides with and is in front of $J$ from $\Sigma$ " iff $\operatorname{ext}(I) e q_{a} \operatorname{ext}(J)$ and $\ell(I)>\ell(J)$;

- $I c^{-} J: \Sigma$, read as " $I$ coincides with $J$ and $J$ is in front of $I$ from $\Sigma$ " iff $\operatorname{ext}(I) e q_{a} \operatorname{ext}(J)$ and $\ell(I)<$ $\ell(J)$;

There is also a reflexive, symmetric and transitive relation $e q, I$ eq $J: \Sigma$, read as " $I$ is equal to $J$ from $\Sigma$ " that only holds if $I=J$ (i.e. $\operatorname{ext}(I) e q_{a} \operatorname{ext}(J)$ and $\ell(I)=\ell(J)$ ). Note that the information about layers is only effectively used when the related objects are in an occlusion state.

The notation “: $\Sigma$ " specifying the viewpoints in IOC relations (e.g. $I R J: \Sigma$, for $R$ an IOC relation) will be omitted whenever the viewpoint is clear from the context.

The set of basic relations of the Interval Occlusion Calculus defines the transition network represented in Figure 5.

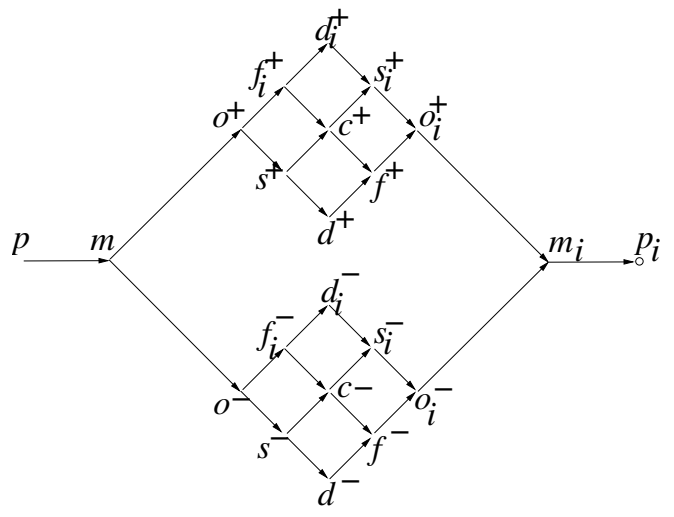

Fig. 5. Interval Occlusion Calculus transition network.

The composition of IOC relations, considering a single viewpoint, is defined as follows.

Definition 1 (Composition of IOC relations). Let a basic relation in IOC be either $\alpha, \beta, \alpha^{+}, \beta^{+}, \alpha^{-}$or $\beta^{-}$, for a given viewpoint, and let $\alpha_{a}$ and $\beta_{a}$ denote the related Allen basic relation (for instance, $\alpha_{a}$ is $\alpha$, or $\alpha^{+}$or $\alpha^{-}$without the layer information). Then, composition in IOC is defined by the 
following cases, where the composition in the right-hand side of the formulae below (denoted by $\circ_{a}$ ) refers to composition in Allen's algebra:

- $\alpha^{+} \circ \beta^{+}=\left(\alpha_{a} \circ_{a} \beta_{a}\right)^{+} ;$

- $\alpha^{-} \circ \beta^{-}=\left(\alpha_{a} \circ_{a} \beta_{a}\right)^{-}$;

- $\quad \alpha \circ \beta, \alpha^{+} \circ \beta^{-}$and $\alpha^{-} \circ \beta^{+}=\left\{\left(\alpha_{a} \circ_{a} \beta_{a}\right)^{+} \cup\left(\alpha_{a} \circ_{a}\right.\right.$ $\left.\left.\beta_{a}\right)^{-} \cup\{e q\}\right\}$ if $\beta$ is the inverse of $\alpha$, and $\left\{\left(\alpha_{a} \circ_{a}\right.\right.$ $\left.\left.\beta_{a}\right)^{+} \cup\left(\alpha_{a} \circ_{a} \beta_{a}\right)^{-}\right\}$otherwise.

From the basic relations above, it is possible to define total occlusion as the disjunction of starts, during, finishes and coincides (as stated in Definition 2 below). In the remainder of this paper we use the notation $a\left\{r_{1}, r_{2} \ldots, r_{n}\right\} b$ (for intervals $a$ and $b$ and relations $r_{i}$ ) as an abbreviation to the disjunction of relations between $a$ and $b$.

Definition 2 (Total Occlusion). Let $A$ and $B$ be two objects, $\Sigma a$ viewpoint and $a$ and $b$ two intervals such that $a=$ $\operatorname{image}(A, \Sigma)$ and $b=\operatorname{image}(B, \Sigma)$, we define the relation "totally occludes" (to) as

- $A$ to ${ }^{+} B: \Sigma$, read as “A totally occludes B from $\Sigma$ ” iff $a\left\{s_{i}^{+}, d_{i}^{+}, f_{i}^{+}, c^{+}\right\} b$.

- $A$ to ${ }^{-} B: \Sigma$, read as “ $A$ is totally occluded by $B$ from $\Sigma$ ” iff $a\left\{s^{-}, d^{-}, f^{-}, c^{-}\right\} b$.

In the remainder of this paper, whenever observations from multiple agents are compared, we assume that they related to the same time instant. The definition of a full spatio-temporal version of IOC is still work in progress.

Definition 3 (is_Behind). Let $A$ be an object, $\Sigma_{i}$ a viewpoint and $\Sigma_{x}$ some viewpoint (such that $\Sigma_{i} \neq \Sigma_{x}$ ). Let also image $\left(A, \Sigma_{x}\right)$ be an interval related to the observation of $A$ by $\Sigma_{x}$. We say that: $A$ is_behind $\Sigma_{i}$ read as " $A$ is behind $\Sigma_{i}$ " if the following conditions are met:

- there is no object o $(o \neq A)$ such that "o to ${ }^{+} A$ " from $\Sigma_{i}$; and

- $\quad$ image $\left(A, \Sigma_{i}\right)$ does not occur as an argument in any relation observed by $\Sigma_{i}$; and

- $\quad$ there is a $\Sigma_{j}\left(\Sigma_{j} \neq \Sigma_{i}\right)$ such that image $\left(A, \Sigma_{j}\right)$ and image $\left(\Sigma_{i}, \Sigma_{j}\right)$ occur as arguments of some relations observed by $\Sigma_{j}$.

It is worth pointing out that Definition 3 is not a necessary condition for $i s \_B e h i n d$ as the general concept holds independently of the existence of observers. However, Definition 3 can be used when reasoning about multiple viewpoints.

Theorem 1. If there is a viewpoint $\Sigma_{i}$ such that for some object $A$ and a viewpoint $\Sigma_{j}\left(\Sigma_{j} \neq \Sigma_{i}\right)$, image $\left(A, \Sigma_{i}\right)$ does not occur as argument in any relation observed by $\Sigma_{i}$ but both image $\left(A, \Sigma_{j}\right)$ and image $\left(\Sigma_{i}, \Sigma_{j}\right)$ occur as arguments in some relations observed by $\Sigma_{j}$ then $A$ is occluded or is behind $\Sigma_{i}$.

Proof: For the purpose of reductio ad absurdum, let's assume that $A$ is seen from $\Sigma_{j}$ but not from $\Sigma_{i}$ and that there is an IOC relation about $\operatorname{image}\left(A, \Sigma_{i}\right)$ in the set of
TABLE I. A PORTION OF THE IOC TRANSLATION TABLE FOR THE SITUATION SHOWN IN FIG. 6; "n.d." IN THE TABLE MEANS non defined

\begin{tabular}{|c|c|c|c|}
\hline & $\sigma_{2} p b$ & $\sigma_{2} p_{i} b$ & $\sigma_{2} s^{+} b$ \\
\hline$\sigma_{2} p a$ & $\begin{array}{c}a^{\prime}\left\{p, m, o^{+}, s^{+}, c^{+}, d_{i}^{+}\right. \\
\left.d^{+}, f^{+}, o_{i}^{+}, m_{i}, p_{i}\right\} b^{\prime}\end{array}$ & $n . d$. & $n . d$. \\
\hline$\sigma_{2} p_{i} a$ & $a^{\prime}\left\{p, p_{i}\right\} b^{\prime}$ & $\begin{array}{c}a^{\prime}\left\{p, m, o^{-}\right. \\
\left.s^{-}, d^{-}\right\} b^{\prime}\end{array}$ & $\left\{a^{\prime} p b^{\prime}\right\}$ \\
\hline$\sigma_{2} m a$ & $\begin{array}{c}a^{\prime}\left\{p, m, o^{+}, s^{+}, c^{+}, d_{i}^{+}\right. \\
\left.d^{+}, f^{+}, o_{i}^{+}, m_{i}, p_{i}\right\} b^{\prime}\end{array}$ & $n . d$. & $n . d$. \\
\hline$\sigma_{2} o^{+} a$ & $\begin{array}{c}a^{\prime}\left\{p, m, o^{+}, s^{+}, c^{+}, d_{i}^{+}\right. \\
\left.d^{+}, f^{+}, o_{i}^{+}, m_{i}, p_{i}\right\} b^{\prime}\end{array}$ & $n . d$. & $n . d$. \\
\hline$\sigma_{2} s^{+} a$ & $a^{\prime}\left\{p, m, o^{+}\right\} b^{\prime}$ & $n . d$. & $n . d$. \\
\hline
\end{tabular}

observations of $\Sigma_{i}$ that is not one of the set $\left\{o^{k}, s^{k}, d^{k}, f^{k}, c^{k}\right\}$ $(k \in\{+,-\})$, i.e., informally, it is not hidden from $\Sigma_{i}$, then $A$ should be visible from $\Sigma_{i}$.

It is worth noting that, without having a second observation from $\Sigma_{j}$ in Theorem $1, \Sigma_{i}$ might assume that the object $A$ is non-existent, or outside its field of view. Theorem 1 is an example of a commonsense fact that follows as a consequence of a mathematical formalism.

\section{A. IOC translation table}

From the diagram shown in Fig. 4 it is possible to define the translation from the observations of one agent to another agent's viewpoint.

This translation can be encoded by a table (called the IOC translation table) that is defined as follows. Let $\mathcal{S}$ be the set of IOC relations, $\Sigma_{1}$ and $\Sigma_{2}$ be two viewpoints observing two distinct objects $A$ and $B$, such that $a=\operatorname{image}\left(A, \Sigma_{1}\right), b=$ $\operatorname{image}\left(B, \Sigma_{1}\right), \sigma_{2}=\operatorname{image}\left(\Sigma_{2}, \Sigma_{1}\right), a^{\prime}=\operatorname{image}\left(A, \Sigma_{2}\right)$, $b^{\prime}=\operatorname{image}\left(B, \Sigma_{2}\right)$ and $\sigma_{1}=\operatorname{image}\left(\Sigma_{1}, \Sigma_{2}\right)$. Assuming that $\Sigma_{1}$ 's location with respect to the objects $A$ and $B$ is known and from that point it observes the relations $R_{i}, R_{j}$ and $R_{k}$ between $A, B$, and $\Sigma_{2}$ (respectively, $a R_{i} b, a R_{j} \sigma_{2}$, and $\left\{b R_{k} \sigma_{2}\right\}$, for $\left\{R_{i}, R_{j}, R_{k}\right\} \in \mathcal{S}$ ) then the translation table gives the set of possible relations $\{R\}$ between $A$ and $B$ as observed from $\Sigma_{2}$, i.e. $\left\{a^{\prime}\{R\} b^{\prime}\right\}$. Note that this is a table representing a function with three arguments: $R_{i}(a, b), R_{j}\left(a, \sigma_{2}\right), R_{k}\left(b, \sigma_{2}\right)$.

The translation table can be built in the following way. For every location of an observer $\Sigma_{1}$ around a pair of objects $A$ and $B$ ( $c f$. Fig. 4 ), given that $\Sigma_{1}$ also views another observer $\Sigma_{2}$ (i.e. $\sigma_{2}$ appears in some IOC relation with respect to $a$ and $b$ as observed by $\Sigma_{1}$ ) the table is built by considering exhaustively all the possible locations of $\Sigma_{2}$, excluding those that are inconsistent with $\Sigma_{1}$ 's observations. Note that the locations of a viewpoint are directly related to its possible observations.

Table I shows part of the translation table for an observer $\Sigma_{1}$ located in the region where it observes $a p b$ (Fig. 6). The entire table, in this case, has size $22 \times 22$.

Figure 6 illustrates the construction of Table I. In this figure, considering the lines of sight between $\Sigma_{1}, A$ and $B$, there are five regions to consider: Regions (1), (2) and (3) and the two red-dashed regions: one between $\Sigma_{1}$ and $A$ and another between $\Sigma_{1}$ and $B$.

If $\Sigma_{1}$ observes $a \quad p \quad b$ and $\left\{\sigma_{2}\left\{p, m, o^{+}\right\} a\right\}$, then $\Sigma_{2}$ is in Region (1) and we have necessarily that $\left\{\begin{array}{lll}\sigma_{2} & p & b\end{array}\right\}$ (note the symbol "n.d." - non defined- in the related table entries in Table I). Since, in this situation, $\Sigma_{2}$ can 
be at any point in Region (1) then the set of relations between $A$ and $B$ that can be observed by $\Sigma_{2}$ is $\left\{a^{\prime}\left\{p, m, o^{+}, s^{+}, f^{+}, c^{+}, d_{i}^{+}, d^{+}, o_{i}^{+}, m_{i}, p_{i}\right\} b^{\prime}\right\}$ (i.e., all the possible IOC relations between the images of $A$ and $B$ that are contained in Region (1), $c f$. Fig. 6).

If $\Sigma_{1}$ sees $\left\{\begin{array}{lll}a & p & b\end{array}\right\},\left\{\begin{array}{lll}\sigma_{2} & p_{i} & a\end{array}\right\}$, and $\left\{\begin{array}{lll}\sigma_{2} & p & b\end{array}\right\}$, then $\Sigma_{2}$ is in Region (2). The possible observations of $\Sigma_{2}$ is the set $\left\{a^{\prime}\left\{p_{i}, p\right\} b^{\prime}\right\}$ (cf. $2^{n d}$ line, 1 st column, of Table I).

If $\Sigma_{1}$ sees $\{a p b\},\left\{\sigma_{2} p_{i} a\right\}$, and $\left\{\sigma_{2}\left\{p_{i}, m_{i}, o_{i}^{-}, o_{i}^{+}\right\} b\right\}$, then $\Sigma_{2}$ is located at any point in Region (3). So the entry in the translation table in this case is $\left\{a^{\prime}\left\{p, m, o^{-}, s^{-}, d^{-}\right\} b^{\prime}\right\}$ (the entry for $\left\{\sigma_{2} p_{i} b\right\}$ is in the $2^{\text {nd }}$ column of Table I).

If $\Sigma_{2}$ is in the red-dashed regions in between $A$ and $\Sigma_{1}$, i.e. $\left\{\sigma_{2}\left\{s^{+}, f^{+}, d^{+}\right\} a\right\}$, then the translation would be $\left\{a^{\prime}\left\{p, m, o^{+}\right\} b^{\prime}\right\}$ (shown in the last line of the table). If $\Sigma_{2}$ is in the red-dashed region between $\Sigma_{1}$ and $B$, $\left\{\sigma_{2}\left\{s^{+}, f^{+}, d^{+}\right\} b\right\}$, then the translation would be $\left\{a^{\prime} p b^{\prime}\right\}$ (the entry for $\left\{\sigma_{2} s^{+} b\right\}$ is in the $3^{r d}$ column of Table I).

The reasoning is analogous to all the other possible locations of $\Sigma_{1}$.

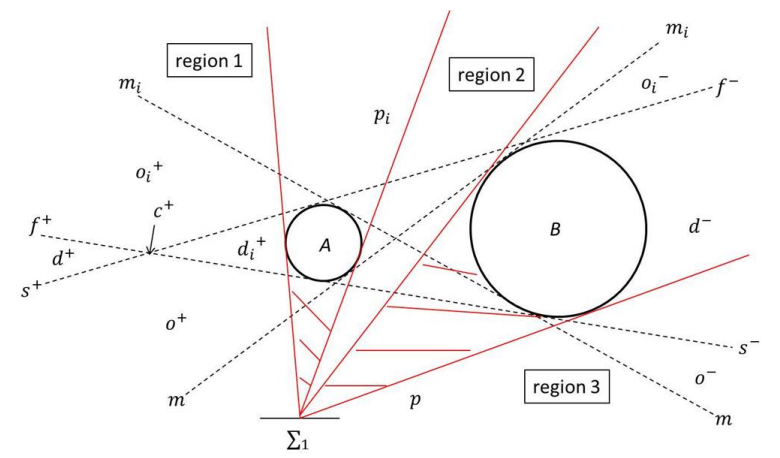

Fig. 6. Example of how to build a translation table.

In order to avoid self-referent descriptions, the observations made by a viewpoint $\Sigma_{j}$ about another viewpoint $\Sigma_{i}$ will not be translated to $\Sigma_{i}$ itself.

It is worth pointing out also that, although the construction of the table assumes that the viewpoints are visible by the agent that receives the translation, its use is not restricted to this case. For instance, in the example shown in Figure $6, \Sigma_{1}$ does not need to see $\Sigma_{2}$ to reason about what the latter would perceive being at a particular region around objects $A$ and $B$, and vice versa.

\section{B. Example}

Let's consider the situation shown in Figure 7, with two objects $C$ and $D$ and two observers $\Sigma_{1}$ and $\Sigma_{2}$.

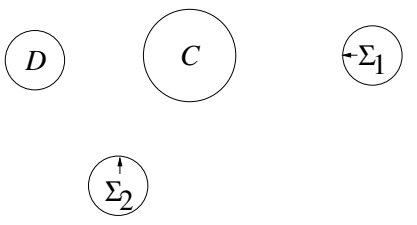

Fig. 7. Objects $C$ and $D$ and viewpoints $\Sigma_{1}$ and $\Sigma_{2}$.
The observations made from viewpoints $\Sigma_{1}$ and $\Sigma_{2}$ are described in the formulae below, where $c=\operatorname{image}\left(C, \Sigma_{1}\right)$, $d=\operatorname{image}\left(D, \Sigma_{1}\right), c^{\prime}=\operatorname{image}\left(C, \Sigma_{2}\right), d^{\prime}=\operatorname{image}\left(D, \Sigma_{2}\right)$, $\sigma_{2}=\operatorname{image}\left(\Sigma_{2}, \Sigma_{1}\right)$ and $\sigma_{1}=\operatorname{image}\left(\Sigma_{1}, \Sigma_{2}\right)$.

$$
\begin{aligned}
& \sigma_{2} p c: \Sigma_{1} \\
& d^{\prime} p c^{\prime}: \Sigma_{2} \\
& c^{\prime} p \sigma_{1}: \Sigma_{2} \\
& d^{\prime} p \sigma_{1}: \Sigma_{2}
\end{aligned}
$$

From Theorem 1 and the fact that $d^{\prime}$ and $\sigma_{1}$ are in the argument of the relations observed by $\Sigma_{2}$, but $d$ is not an argument of a relation observed by $\Sigma_{1}$, we have:

$$
\begin{aligned}
& c\left\{c^{+}, f^{+}, s^{+}, d^{+}\right\} d: \Sigma_{1} \\
& \text { (i.e. cto } \left.{ }^{+} d: \Sigma_{1}\right) .
\end{aligned}
$$

By using the translation table (Table I) to translate what $\Sigma_{2}$ perceives in terms of $\Sigma_{1}$ 's point of view, we get:

$$
c\left\{p, m, o^{+}, t o^{+}, o_{i}^{+}, p_{i}, m_{i}\right\} d: \Sigma_{1}
$$

which is consistent with the fact that $c t o^{+} d: \Sigma_{1}$ as given in Formula 5.

\section{BASIC ALGORITHM}

The example described in Section V-B illustrates the following basic algorithm for verifying consistency in IOC:

1) pick a viewpoint $\Sigma_{i}$ from the set of observers in a domain;

2) translate the observations of other viewpoints $\Sigma_{j}$ present in the domain $\left(\Sigma_{j} \neq \Sigma_{i}\right)$ to $\Sigma_{i}$ 's perspective (as described in Section V-A);

3) let $\mathcal{S}$ be the union of $\Sigma_{i}$ 's observations with the translated observations from other viewpoints (as obtained in step 2);

4) enforce consistency of the set of relations $\mathcal{S}$ by algebraic closure, as described in Section III.

\section{The IOC IS A Relation Algebra}

In this section we prove that IOC is a relation algebra. This result implies that IOC has a well-founded semantics and that it can be used as an algebraic tool for representing and operating on spatial knowledge. In order to prove that IOC is a relation algebra, we need to prove that its composition is associative.

Theorem 2. The algebra of the Interval Occlusion Calculus is a relation algebra.

Proof: Let $\alpha, \beta$ and $\gamma$ be IOC relations, and $\alpha_{a}, \beta_{a}$ and $\gamma_{a}$ their related Allen's basic relations (i.e. $\alpha, \beta$ and $\gamma$ without the layer information). Let also $\circ$ be the composition in IOC and $\circ_{a}$ the composition in Allen's Algebra. In this proof we use the suffix $I$ to represent the inverse of a relation (e.g. $\alpha I$ is the inverse of $\alpha$ ).

Consider a typical non-trivial case: $\left(\alpha^{+} \circ \beta^{+}\right) \circ \gamma^{-}$. We must show that this is equal to $\alpha^{+} \circ\left(\beta^{+} \circ \gamma^{-}\right)$.

According to the explicit definition of the composition of IOC relations (Definition 1), we have 
$\left(\alpha^{+} \circ \beta^{+}\right) \circ \gamma^{-}=\left(\alpha_{a} \circ \circ_{a} \beta_{a}\right)^{+} \circ \gamma$

$=\left(\alpha_{a} \circ_{a} \beta_{a} \circ_{a} \gamma_{a}\right)^{+} \cup\left(\alpha_{a} \circ_{a} \beta_{a} \circ_{a} \gamma_{a}\right)^{-}$if $\gamma I \notin(\alpha \circ \beta)$,

or $\left(\alpha_{a} \circ_{a} \beta_{a} \circ_{a} \gamma_{a}\right)^{+} \cup\left(\alpha_{a} \circ_{a} \beta_{a} \circ_{a} \gamma_{a}\right)^{-} \cup\{e q\}$ otherwise.

On the other hand, $\alpha^{+} \circ\left(\beta^{+} \circ \gamma^{-}\right)=\alpha^{+} \circ\left(\left(\beta_{a} \circ \circ_{a} \gamma_{a}\right)^{+} \cup\right.$ $\left.\left(\beta_{a} \circ_{a} \gamma_{a}\right)^{-}\right)$if $\alpha \notin(\beta \circ \gamma)$, or $\alpha^{+} \circ\left(\left(\beta_{a} \circ_{a} \gamma_{a}\right)^{+} \cup\left(\beta_{a} \circ \circ_{a}\right.\right.$ $\left.\left.\gamma_{a}\right)^{-} \cup\{e q\}\right)$ otherwise; that is

$\left\{\left(\alpha_{a} \circ_{a} \beta_{a} \circ_{a} \gamma_{a}\right)^{+} \cup\left(\alpha_{a} \circ_{a} \beta_{a} \circ_{a} \gamma_{a}\right)^{-}\right\}$if $\alpha I \notin(\beta \circ \gamma)$, or $\left\{\left(\alpha_{a} \circ_{a} \beta_{a} \circ_{a} \gamma_{a}\right)^{+} \cup\left(\alpha_{a} \circ_{a} \beta_{a} \circ_{a} \gamma_{a}\right)^{-} \cup\{e q\}\right\}$ otherwise.

Now since the Allen's Interval algebra is a relation algebra, it satisfies the well-known axiom asserting that the conditions $\gamma_{a} I \notin\left(\alpha_{a} \circ_{a} \beta_{a}\right)$ and $\alpha_{a} I \notin\left(\beta_{a} \circ_{a} \gamma_{a}\right)$ are equivalent.

This proof also holds for the POC (presented in Section II), and for occlusion algebras based on other relation algebras.

\section{RUNNING-TIME COMPLEXITY}

Sound and complete consistency checking for Allen's Algebra is NP complete [12]. As IOC is an extension of Allen's Algebra, consistency checking in IOC falls within the NP-hard class of problems. However, [12] shows that path consistency provides a sound cubic-time method for consistency checking in Allen's Algebra.

In a typical situation, observers provide descriptions using only the basic elements of the IOC. In case of occlusion, disjunctive relations such as $\left\{s^{k}, d^{k}, f^{k}, c^{k}\right\}$ (for $k \in\{+,-\}$ ) express total occlusion. Thus:

Theorem 3. Sound (but not complete) consistency checking on an atomic network of IOC relations can be solved in polynomial time with respect to the number of objects observed.

Proof:

1) Consider the IOC network obtained by ignoring the layer information. This is reduced to the original Allen's Calculus and, therefore, path consistency provides a cubic time sound method for consistency checking.

2) Now consider the layer information. A relation such as $p, p_{i}, m, m_{i}$ results in the universal constraint for the layer. Other relations imply the basic relations of the Point Algebra. Thus, consistency can be solved in cubic time by algebraic closure [2].

This complexity result applies to reasoning about multiple viewpoints, since (in this work) the information from the various viewpoints is translated to the viewpoint of a single agent (as described in Section V-A). For a given viewpoint, the translation process is a table lookup procedure $(\mathcal{O}(1))$ applied to every other viewpoint in the scene. Thus, the translation process runs in linear time with respect to the number of viewpoints in the scene.

\section{CONCLUSION}

This paper is concerned with two issues: occlusion and multiple viewpoints. Occlusion prevents sensors from assessing part of the objects present in a scene. Multiple viewpoints result in distinct observers of the same scene providing distinct descriptions of what they observe.
To the best of our knowledge, the present paper is the first to define an extension of the Allen's Interval Algebra (which we called Interval Occlusion Calculus - IOC) to provide a rigorous account for occlusion and multiple viewpoints. Occlusion, in this context, is represented as relations within a layered set of intervals. The fusion of the distinct scene observations from multiple agents is dealt with by means of explicit rules for translating the observations from one point of view to another. These observations are used for patching up the local descriptions of a scene. On a real application, IOC shall provide the consistency of all the observations from the multi-agent domain. It should allow for the identification of wrong observations, from faulty sensors for instance.

As in this work we are mostly interested in formalising spatial interposition (or occlusion), the relative sizes of the intervals were abstracted away. The interval sizes could be considered by using the INDU calculus [13], instead of Allen's algebra, as the underlying theory. This issue is left for further research.

\section{Acknowledgements}

Paulo E. Santos acknowledges support from FAPESP (grant 2012/04089-3) and CNPq (grant PQ2-307093/2014-0).

\section{REFERENCES}

[1] A. G. Cohn and J. Renz, "Qualitative spatial reasoning," in Handbook of Knowledge Representation, F. van Harmelen, V. Lifschitz, and B. Porter, Eds. Elsevier, 2007, pp. 551-596.

[2] G. Ligozat, Qualitative Spatial and Temporal Reasoning. Wiley, 2011.

[3] D. Randell, M. Witkowski, and M. Shanahan, "From images to bodies: Modeling and exploiting spatial occlusion and motion parallax," in Proc. of IJCAI, Seattle, U.S., 2001, pp. 57-63.

[4] J. F. Allen, "Maintaining knowledge about temporal intervals," Commun. ACM, vol. 26, no. 11, pp. 832-843, Nov. 1983.

[5] A. P. Petrov and L. V. Kuzmin, "Visual space geometry derived from occlusion axioms." Journal of Mathematical Imaging and Vision, vol. 6, no. 2-3, pp. 291-308, 1996

[6] A. Galton, "Lines of sight," in Proc. of the Seventh Annual Conference of AI and Cognitive Science, Dublin, Ireland, 1994, pp. 103-113.

[7] R. Villemaire and S. Hall, "Reasoning about visibility," Journal of Applied Logic, vol. 10, no. 2, pp. 163 - 178, 2012.

[8] P. Guha, A. Mukerjee, and K. S. Venkatesh, "OCS-14: You can get occluded in fourteen ways," in Proc. of IJCAI, 2011, pp. 1665-1670.

[9] C. Kray, "The benefits of multi-agent systems in spatial reasoning," in Proceedings of the Fourteenth International Florida Artificial Intelligence Research Society Conference. AAAI Press, 2001, pp. 552-556.

[10] N. I. Karacapilidis, D. Papadias, and M. J. Egenhofer, "Collaborative spatial decision making with qualitative constraints," in ACM-GIS, 1995, pp. 53-59.

[11] G. Edwards and G. Ligozat, "A formal model for structuring local perceptions of environmental space," Cognitive Processing, vol. 5, no. 1, pp. 3-9, 2004.

[12] A. Gerevini, "Chapter 8 processing qualitative temporal constraints," Foundations of Artificial Intelligence, vol. 1, pp. 247-276, 2005.

[13] A. K. Pujari, G. V. Kumari, and A. Sattar, "Indu: An interval and duration network," in Australian Joint Conference on Artificial Intelligence, 1999, pp. 291-303. 